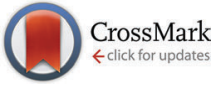

Cite this: Chem. Commun., 2015, 51, 12958

Received 3rd March 2015, Accepted 6th July 2015

DOI: $10.1039 / c 5 c c 01823 d$

www.rsc.org/chemcomm

\section{Strong antiferromagnetic exchange between manganese phthalocyanine and ferromagnetic europium oxide $\dagger$}

\author{
Christian Wäckerlin, ${ }^{* a}$ Fabio Donati, ${ }^{a}$ Aparajita Singha, ${ }^{a}$ Romana Baltic, ${ }^{a}$ \\ Anne-Christine Uldry, ${ }^{\mathrm{b}}$ Bernard Delley, ${ }^{\mathrm{b}}$ Stefano Rusponi ${ }^{\mathrm{a}}$ and Jan Dreiser*ac
}

\begin{abstract}
We report on the antiferromagnetic exchange coupling between a submonolayer of $\mathrm{Mn}(\mathrm{II})$-phthalocyanine molecules and a ferromagnetic Eu(II)-oxide thin film. The exchange energy is larger by nearly two orders of magnitude compared to previous studies involving oxidic substrates.
\end{abstract}

Within the quest of organic spintronics ${ }^{1}$ it is important to interface magnetic molecules with inorganic spin injection materials serving as electrodes. ${ }^{2}$ Spin injection through the ferromagnetic (FM) semiconductor europium(II)-oxide (EuO) with spin polarization of almost $100 \%{ }^{3}$ is more promising than from FM metals ${ }^{4}$ in which the spin polarization is on the order of only $50 \%$. Yet, the electronic configuration and magnetic properties of molecules adsorbed at the surface of FM semiconducting oxides remain largely unexplored despite their fundamental relevance ${ }^{1 c}$ and are in contrast to the wealth of reports focusing on the magnetic properties of paramagnetic organic semiconductors at the interface to FM metals. ${ }^{5}$ Only in the case of a $\mathrm{Cu}(\mathrm{II})$ complex adsorbed on ferrimagnetic magnetite $\left(\mathrm{Fe}_{3} \mathrm{O}_{4}\right)$ a weak exchange interaction was observed, ${ }^{6}$ while for the $\mathrm{TbPc}_{2}$ single-molecule magnet deposited on the FM semiconductor $\mathrm{La}_{0.3} \mathrm{Sr}_{0.7} \mathrm{MnO}_{3}$ (LSMO) the magnetic interaction was undetectably small. ${ }^{7}$

Here we show that $\mathrm{Mn}(\mathrm{II})$-phthalocyanine (MnPc) couples antiferromagnetically to a thin film of the FM semiconductor $\mathrm{Eu}(\mathrm{II})$-oxide ${ }^{4 b, 8}$ grown on highly oriented pyrolytic graphite (HOPG). Our experimental data supported by multiplet calculations reveal that the molecules adsorb with their macrocycles parallel to the surface and that the surface-adsorbed MnPc exhibits a high-spin $S=5 / 2$ state.

\footnotetext{
${ }^{a}$ Institute of Condensed Matter Physics, Ecole Polytechnique Fédérale de Lausanne, CH-1015 Lausanne, Switzerland. E-mail: christian@waeckerlin.com

${ }^{b}$ Condensed Matter Theory, Paul Scherrer Institut, CH-5232 Villigen, Switzerland

${ }^{c}$ Swiss Light Source, Paul Scherrer Institut, CH-5232 Villigen PSI, Switzerland.

E-mail: jan.dreiser@psi.ch

$\dagger$ Electronic supplementary information (ESI) available: Sample preparation, experimental details of XAS, additional multiplet calculations, magnetic moments of Mn and $\mathrm{Eu}$ and sum-rule correction factors, $\mathrm{X}$-ray spectra at $50 \mathrm{~K}$, at $110 \mathrm{~K}$, and on $\mathrm{EuO}_{1+x}$, and the Brillouin function model. See DOI: 10.1039/c5ec01823d
}

a
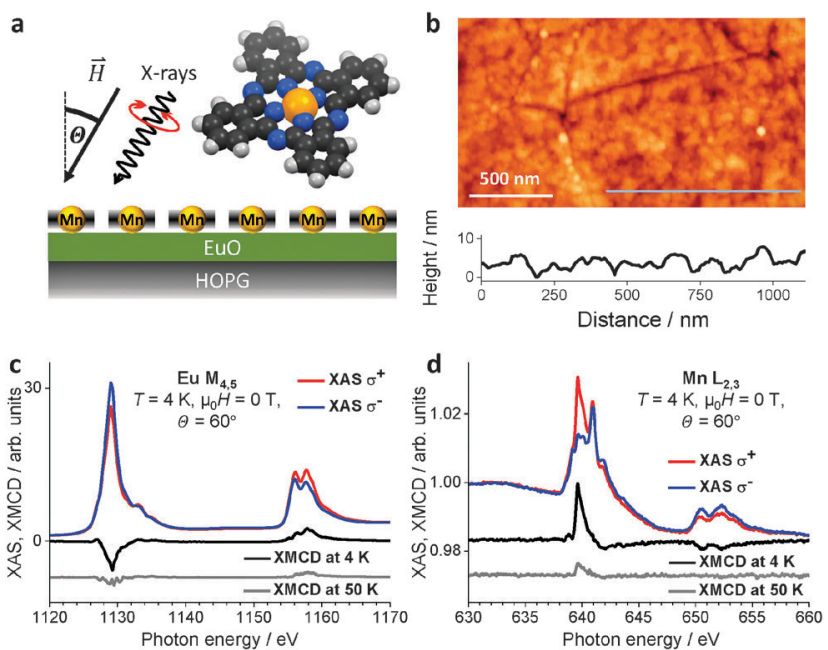

Fig. 1 (a) Structure of $\mathrm{MnPc}$ and sketch of the investigated sample. The magnetic field and the X-ray beam were kept parallel. (b) Atomic force microscope (ambient conditions) image of the EuO thin film with the line profile. The height difference between the brightest and darkest areas is $20 \mathrm{~nm}$. (c and d) XAS and XMCD recorded in remanence at the Eu $M_{4,5}$ $(3 d \rightarrow 4 f)$ and $M n L_{2,3}(2 p \rightarrow 3 d)$ edges. The $X M C D$ spectra are shown with an offset. The data evidence a net remanent $\mathrm{Mn}$ magnetic moment, revealing that MnPc couples antiferromagnetically to the ferromagnetic EuO thin film.

A sketch of the sample structure and an atomic force microscope image of the bare EuO surface are shown in Fig. 1a and b. The Eu(II)-oxide thin film was grown in ultra-high vacuum by reactive molecular beam epitaxy ${ }^{8 c}$ and, subsequently, a submonolayer amount of MnPc was deposited ( $c f$. ESI $\dagger$ ).

Grazing-incidence X-ray absorption spectra (XAS) and X-ray magnetic circular dichroism (XMCD) recorded at the $\mathrm{Eu} \mathrm{M}_{4,5}$ and $\mathrm{Mn} \mathrm{L}_{2,3}$ edges at 4 and $50 \mathrm{~K}$ in remanence are plotted in Fig. $1 \mathrm{c}$ and d. The remanent XMCD signal at the Eu $\mathrm{M}_{4,5}$ edge confirms the ferromagnetism of the EuO thin film. ${ }^{8 a}$ The Mn $\mathrm{L}_{2,3}$ spectra exhibit a strong XMCD signal with opposite sign demonstrating antiparallel alignment of the MnPc and $\mathrm{EuO}$ magnetization indicating antiferromagnetic (AFM) coupling of the MnPc molecules to the FM EuO substrate. 
We have performed effective point-charge atomic multiplet calculations using the MultiX ${ }^{9}$ software to determine the adsorption geometry of MnPc and to shed light on the Mn and Eu electronic ground states and magnetic moments. This powerful approach is highly useful for the description of the low-symmetry ligand fields present in surface-adsorbed organometallic complexes. Least-squares fits optimizing the strengths and positions of the effective point charges to reproduce the experimental Mn circular and linear dichroism spectra allow us to extract the sought information from the experimental spectra.

Grazing-incidence X-ray spectra recorded at the $\mathrm{Eu} \mathrm{M}_{4,5}$ edges at $6.8 \mathrm{~T}$ and $4 \mathrm{~K}$ are shown in Fig. $2 \mathrm{a}$. The calculated spectra for $\mathrm{Eu}(\mathrm{II})$ considering only the first coordination sphere, that is, taking into account the closest oxygen atoms, are in excellent agreement with the experimental data. The comparison of the calculated spectra for $\mathrm{Eu}(\mathrm{III})$ with literature spectra ${ }^{10}$ reveals that the nonmagnetic $\mathrm{Eu}_{2} \mathrm{O}_{3}$ is virtually absent.

The $\mathrm{Mn}_{2,3}$ XAS and XMCD spectra recorded at $6.8 \mathrm{~T}$ and $4 \mathrm{~K}$ in grazing incidence, the X-ray natural linear dichroism (XNLD) spectra and the corresponding calculated spectra are presented in Fig. $2 \mathrm{~b}-\mathrm{d}$. The Mn spectra differ significantly from bulk MnPc, ${ }^{11}$ e.g., the $\mathrm{L}_{3}$ and $\mathrm{L}_{2}$ XMCD signals are antiparallel while in the bulk they are parallel indicating a large difference in the $\mathrm{Mn}$ electronic structure. Notably, the present spectra are also different from those of MnPc adsorbed on $\mathrm{Co}$ and $\mathrm{Cu}$ transition metal surfaces ${ }^{5 e f}$ and on an oxygen reconstructed Co surface. ${ }^{5 h}$ The X-ray spectra exhibit a well resolved substructure consistent with a low molecule-substrate hybridization. ${ }^{5 e}$ The point charges used as an input for the multiplet calculations (Table S1, ESI $\dagger$ ) were optimized in order to minimize the error calculated by the sum of squared deviations between the calculated and experimental spectra, respectively, shown in Fig. 2b-d. Details are given in the ESI. $\uparrow$ The calculations reveal that the
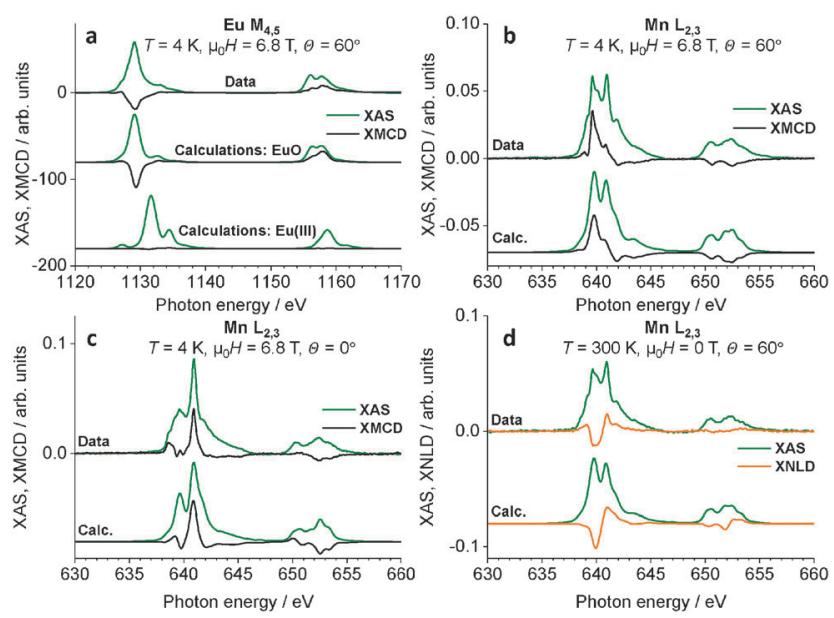

Fig. 2 XAS, XMCD (a-c) and XNLD (d) spectra recorded at the Eu $M_{4,5}$ ( $3 \mathrm{~d} \rightarrow 4 \mathrm{f})$ and $\mathrm{Mn} \mathrm{L}_{2,3}(2 \mathrm{p} \rightarrow 3 \mathrm{~d})$ edges at the temperatures, magnetic fields and $X$-ray incidence angles indicated in the plots. The experimental data are compared to the calculated spectra which are obtained from multiplet calculations based on the MultiX code. ${ }^{9}$ Least-squares fits to all the $\mathrm{MnX}$-ray spectra indicate that the MnPc molecules adsorb with their macrocycles parallel to the surface. ground-state multiplet is characterized by a high-spin $S=5 / 2$ state of the Mn ion. In contrast, the intermediate $S=3 / 2$ state of $\mathrm{Mn}(\mathrm{II})$ as observed in bulk MnPc, ${ }^{11}$ as well as $\mathrm{Mn}$ (I) and $\mathrm{Mn}$ (III) oxidation states can be excluded because the respective XAS calculations yield the spectra which are incompatible with the experimental data ( $c f$. Fig. S1 and S2, ESI $\dagger$ ). Moreover, the flat orientation of the molecules on the EuO surface, consistent with the strong observed magnetic Mn-Eu coupling, can be directly inferred from the orientation of the point-charge ligand field (Table S1, ESI $\dagger$ ) obtained from the fits to the X-ray spectra.

The spin and orbital magnetic moments obtained from the sum rule ${ }^{12}$ analyses of the $\mathrm{Mn} \mathrm{L}_{2,3}$ and $\mathrm{Eu} \mathrm{M}_{4,5} \mathrm{X}$-ray spectra are presented in Table S4 (ESI $\dagger$ ). Note that the value of the effective spin projection $\left\langle S_{\text {eff } z}\right\rangle$ obtained from the sum rule analysis of the Mn spectra is lowered with respect to that of the true spin $\left\langle S_{z}\right\rangle$ because of mixing between the $2 \mathrm{p}_{3 / 2}$ and $2 \mathrm{p}_{1 / 2}$ components. ${ }^{13 a}$ Moreover, it can be modified by the presence of a finite dipole $\left(T_{z}\right)$ term. These difficulties can be overcome by applying the sum rules to the calculated X-ray spectra for which the $\left\langle S_{z}\right\rangle$ value is known. This approach allows us to extract the correction factors $c=\left\langle S_{\text {eff }, z}\right\rangle /\left\langle S_{z}\right\rangle$ for Mn and Eu (Tables S2 and S3, ESI $\dagger$ ), respectively. The obtained correction factor for $\mathrm{Mn}$ is virtually independent of the X-ray incidence angle $\theta$. It agrees very well with the values found for high-spin $3 \mathrm{~d}^{5} \mathrm{Fe}(\mathrm{III})^{13 b}$ and Mn(II). ${ }^{13 a}$ The sum rule analysis also reveals a small orbital moment $\left\langle L_{z}\right\rangle$ of up to $0.3 \mu_{\mathrm{B}}$ which is not reproduced in the multiplet calculations. We attribute this deviation as well as the small deviation of the calculated linear dichroism from the experimental spectra at $639 \mathrm{eV}$ to subtle charge-transfer effects between the Mn(II) ion and the Pc ligand resulting in a minor contribution of the $\mathrm{Mn}(\mathrm{I})-\mathrm{Pc}^{+}$configuration. ${ }^{13 c}$ This configuration which exhibits parallel alignment of spin and orbital angular momenta is neglected in our calculations. Yet, such charge-transfer effects could contribute, together with the exchange coupling to the $\mathrm{EuO}$ film with in-plane magnetic anisotropy, to a slight lowering of the Mn spin magnetic moment to $3.7 \pm 0.3 \mu_{\mathrm{B}}$ observed in grazing incidence at $4 \mathrm{~K}$, which is below the expected value of $5 \mu_{\mathrm{B}}$. The absorption lengths at the $\mathbf{M}_{4,5}$ edges of bulk rare earths are in the order of $\sim 3-10 \mathrm{~nm},{ }^{14}$ which also holds for EuO. Since this length scale is comparable to the electron escape depth of a few $\mathrm{nm}$ in the corresponding $\mathrm{X}$-ray range, saturation effects in the used total electron yield detection have to be considered. Saturation effects are particularly effective at the Eu $\mathrm{M}_{5}$ edge resulting from a sizeable drop of X-ray intensity within the top surface layers owing to the strong absorption. A simple estimation yields that the Eu orbital magnetic moment extracted by the sum rule analysis vanishes, as would be expected for the $4 \mathrm{f}^{7}$ configuration $\mathrm{Eu}(\mathrm{II})$, when the $\mathrm{M}_{5}$ peak of the more strongly absorbed circular polarization is scaled up by $\sim 10 \%$.

A further important parameter characterizing the $\mathrm{MnPc} / \mathrm{EuO}$ interface is the exchange energy $E_{\text {ex }}$, which refers to the energy needed to reverse the MnPc magnetic moment from the antiparallel to the parallel alignment. Since the Mn magnetic moment remains antiparallel to the EuO magnetization even in the presence of an external field of $6.8 \mathrm{~T}$, the exchange energy 


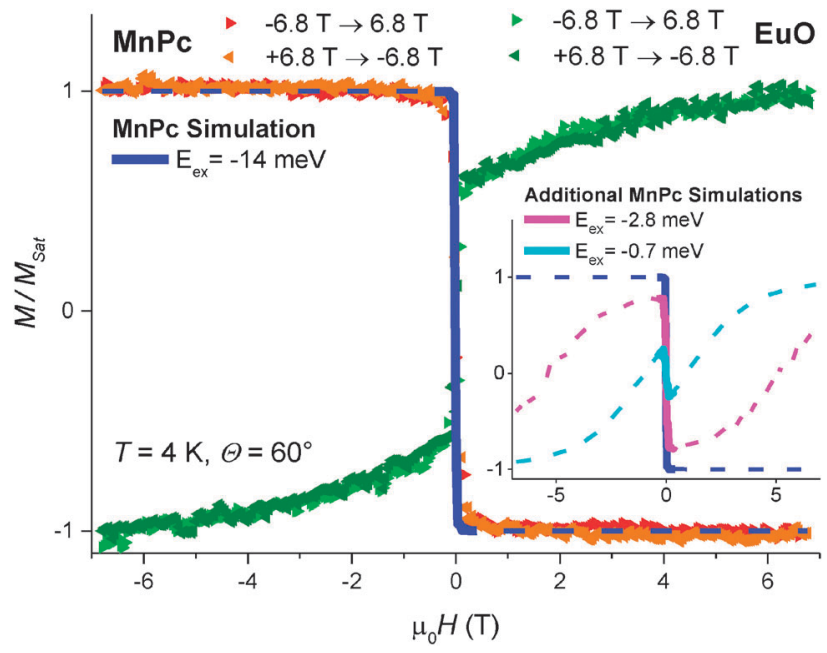

Fig. 3 Normalized magnetization $M(H)$ of the EuO thin film and of the adsorbed MnPc molecules obtained from XMCD. Experimental data and the best-fit curve obtained from the model described in the text are shown as symbols and as a solid line, respectively. Calculated $M n M(H)$ for smaller exchange energies are shown in the inset. The dashed lines denote the field range in which the Eu magnetization is larger by more than $10 \%$ compared to the remanent magnetization, indicating the fields at which anisotropy effects may become relevant. The EuO hysteresis opening of $\sim 12 \mathrm{mT}$ is not resolved. Due to the interaction with the EuO substrate, the magnetization of MnPc saturates already at low magnetic fields.

must be stronger than the corresponding Zeeman splitting of $\sim 4 \mathrm{meV}$. In fact, the magnetization curves $M(H)$ obtained in grazing incidence at $4 \mathrm{~K}$ for both $\mathrm{Mn}$ and $\mathrm{Eu}$ show that the Mn magnetic moment saturates already for $\mu_{0} H>0.5 \mathrm{~T}$ and remains saturated up to $6.8 \mathrm{~T}$ (Fig. 3). The exchange energy can be estimated from the temperature-dependent ratio of the remanent $\mathrm{Mn}$ and $\mathrm{Eu}$ magnetization values (Table S4 and Fig. S4 and S5, ESI $\dagger$ ) by a Brillouin function model (Fig. S7, ESI $\dagger$ ), ${ }^{5 c, d}$ yielding $E_{\mathrm{ex}}^{\mathrm{B}}=-14 \pm 7 \mathrm{meV}$. Furthermore, the magnetic field dependence of the $\mathrm{Mn}$ magnetic moment at $4 \mathrm{~K}$ and the magnetic moment measured at $50 \mathrm{~K}$ can be calculated from the spin Hamiltonian $\hat{\mathcal{H}}=\mu_{0} \mu_{\mathrm{B}} \hat{S}_{\mathrm{Mn}} g_{\mathrm{Mn}}\left(H+H_{\mathrm{ex}}\right)$ with $H_{\mathrm{ex}}=$ $k_{\mathrm{ex}} M_{\mathrm{Eu}}$. Here, $\hat{S}_{\mathrm{Mn}}$ is the Mn spin operator and $g_{\mathrm{Mn}}=2$ is the Mn $g$-factor. The coupling to the FM EuO substrate is taken into account via the effective exchange field $H_{\mathrm{ex}}$ which was set to be proportional to the magnetization $M_{\mathrm{Eu}}$ (in $\mu_{\mathrm{B}}$ ) of the substrate. For $S_{\mathrm{Mn}}=5 / 2$ as found from the X-ray spectra, the exchange energy can be determined from a least-squares fit to be $E_{\mathrm{ex}}^{\mathrm{SH}}=$ $2 g_{\mathrm{Mn}} S_{\mathrm{Mn}} \mu_{0} \mu_{\mathrm{B}} H_{\mathrm{ex}}=-14 \pm 4 \mathrm{meV}$, in excellent agreement with the previous Brillouin function estimation. Magnetic anisotropies are neglected here in order to avoid overparameterization of the model; however, they may influence the shape of the Mn and Eu $M(H)$ at elevated magnetic fields.

The observed exchange energy is two orders of magnitude above the value found for $\operatorname{Er}(\mathrm{III})$ single-ion magnets on $\mathrm{Ni},{ }^{5 k}$ one order of magnitude above the exchange coupling strength of Co-porphyrin on graphene on $\mathrm{Ni}^{5 j}$ and of $\mathrm{TbPc}_{2}$ on $\mathrm{Ni},{ }^{15}$ but it is up to one order of magnitude below the exchange coupling strength of $3 \mathrm{~d}$ transition metal complexes on Co and $\mathrm{Ni}^{5 c, f}$ Remarkably, the $\mathrm{Mn}-\mathrm{Eu}$ magnetic coupling reported here is nearly two orders of magnitude larger than that found for a $\mathrm{Cu}$ porphyrin derivative on magnetite. ${ }^{6}$ The $\mathrm{Mn}-\mathrm{Eu}$ magnetic coupling is likely to be mediated via the Eu $5 \mathrm{~d}$ bands which have been shown to be spin polarized. ${ }^{16 a}$ Because of an AFM coupling between Mn $3 d$ and Eu $5 d$ electrons ${ }^{16 b}$ and the FM coupling between the $\mathrm{Eu} 4 \mathrm{f}$ and $5 \mathrm{~d}$ orbitals, ${ }^{8 a, 16 b}$ the net Mn-Eu coupling is AFM with an exchange coupling strength of $j=E_{\mathrm{ex}} /\left(2 S_{\mathrm{Eu}} S_{\mathrm{Mn}}\right) \cong-0.82 \mathrm{meV}$ taking into account $S_{\mathrm{Eu}}=7 / 2$. The strength of the coupling matches very well with the typical values for $3 \mathrm{~d}-4 \mathrm{f}$ systems. ${ }^{16 b} 3 \mathrm{~d}-4 \mathrm{f}$ super exchange via $\mathrm{O}$ or $\mathrm{N}$ forming a $90^{\circ}$ pathway is unlikely because it is expected to be weak and FM. ${ }^{16 c}$ The estimated Eu-Mn dipolar coupling of $j_{\text {dipolar }} \cong-0.8 \mu \mathrm{eV}$ is far too small to account for the observed coupling strength.

In conclusion, we demonstrate the presence of a strong antiferromagnetic exchange interaction at the interface between the paramagnetic organic semiconductor MnPc and the ferromagnetic semiconductor Eu(II)-oxide. Moreover, the X-ray absorption spectra reveal that the MnPc molecules adsorb flat on the EuO surface. The electronic configuration of $\mathrm{Mn}$ (II) in MnPc is significantly modified upon adsorption on EuO compared to bulk MnPc, leading to a high-spin $S=5 / 2$ state instead of intermediate spin $S=3 / 2$. Our results pave the way for a new class of molecule/ferromagnetic oxide spin interfaces and hybrid molecule/inorganic tunneling spintronic devices.

The X-ray measurements were performed at the EPFL/PSI $X$-Treme beamline ${ }^{17}$ at the Swiss Light Source, Paul Scherrer Institut, Villigen, Switzerland. Rolf Schelldorfer and Prof. T. Jung are kindly acknowledged for the atomic force microscopy images recorded at the Scanning Probe Microscopy User Lab, Laboratory for Micro and Nanotechnology, Paul Scherrer Institut, Villigen, Switzerland. J.D. and C.W. gratefully acknowledge funding from the Swiss National Science Foundation (grant no. PZ00P2_142474). All authors thank Prof. Harald Brune for his kind support.

\section{Notes and references}

1 (a) S. Sanvito, Chem. Soc. Rev., 2011, 40, 3336; (b) V. A. Dediu, L. E. Hueso, I. Bergenti and C. Taliani, Nat. Mater., 2009, 8, 707-716; (c) C. Barraud, P. Seneor, R. Mattana, S. Fusil, K. Bouzehouane, C. Deranlot, P. Graziosi, L. Hueso, I. Bergenti, V. Dediu, F. Petroff and A. Fert, Nat. Phys., 2010, 6, 615-620.

2 M. Cinchetti, K. Heimer, J.-P. Wüstenberg, O. Andreyev, M. Bauer, S. Lach, C. Ziegler, Y. Gao and M. Aeschlimann, Nat. Mater., 2008, 8, 115-119.

3 (a) A. Schmehl, V. Vaithyanathan, A. Herrnberger, S. Thiel, C. Richter, M. Liberati, T. Heeg, M. Röckerath, L. F. Kourkoutis, S. Mühlbauer, P. Böni, D. A. Muller, Y. Barash, J. Schubert, Y. Idzerda, J. Mannhart and D. G. Schlom, Nat. Mater., 2007, 6, 882-887; (b) A. Melville, T. Mairoser, A. Schmehl, D. E. Shai, E. J. Monkman, J. W. Harter, T. Heeg, B. Holländer, J. Schubert, K. M. Shen, J. Mannhart and D. G. Schlom, Appl. Phys. Lett., 2012, 100, 222101.

4 (a) N. Jutong, I. Rungger, C. Schuster, U. Eckern, S. Sanvito and U. Schwingenschlögl, Phys. Rev. B, 2012, 86, 205310; (b) T. Santos and J. Moodera, Phys. Rev. B, 2004, 69, 241203.

5 (a) A. Scheybal, T. Ramsvik, R. Bertschinger, M. Putero, F. Nolting and T. A. Jung, Chem. Phys. Lett., 2005, 411, 214-220; (b) H. Wende, M. Bernien, J. Luo, C. Sorg, N. Ponpandian, J. Kurde, J. Miguel, M. Piantek, X. Xu, P. Eckhold, W. Kuch, K. Baberschke, P. M. Panchmatia, B. Sanyal, P. M. Oppeneer and O. Eriksson, Nat. Mater., 2007, 6, 516-520; (c) M. Bernien, X. Xu, J. Miguel, M. Piantek, P. Eckhold, J. Luo, J. Kurde, W. Kuch, K. Baberschke, H. Wende and P. Srivastava, Phys. Rev. B, 2007, 76, 214406; (d) M. Bernien, J. Miguel, C. Weis, M. E. Ali, 
J. Kurde, B. Krumme, P. M. Panchmatia, B. Sanyal, M. Piantek, P. Srivastava, K. Baberschke, P. M. Oppeneer, O. Eriksson, W. Kuch and H. Wende, Phys. Rev. Lett., 2009, 102, 047202; (e) S. Javaid, M. Bowen, S. Boukari, L. Joly, J.-B. Beaufrand, X. Chen, Y. Dappe, F. Scheurer, J.-P. Kappler, J. Arabski, W. Wulfhekel, M. Alouani and E. Beaurepaire, Phys. Rev. Lett., 2010, 105, 077201; $(f)$ C. Wäckerlin, K. Tarafder, D. Siewert, J. Girovsky, T. Hählen, C. Iacovita, A. Kleibert, F. Nolting, T. A. Jung, P. M. Oppeneer and N. Ballav, Chem. Sci., 2012, 3, 3154-3160; $(g)$ J. Brede, N. Atodiresei, S. Kuck, P. Lazić, V. Caciuc, Y. Morikawa, G. Hoffmann, S. Blügel and R. Wiesendanger, Phys. Rev. Lett., 2010, 105, 047204; (h) C. Wäckerlin, J. Nowakowski, S.-X. Liu, M. Jaggi, D. Siewert, J. Girovsky, A. Shchyrba, T. Hählen, A. Kleibert, P. M. Oppeneer, F. Nolting, S. Decurtins, T. A. Jung and N. Ballav, Adv. Mater., 2013, 25, 2404-2408; (i) E. Annese, F. Casolari, J. Fujii and G. Rossi, Phys. Rev. B, 2013, 87, 054420; $(j)$ C. F. Hermanns, K. Tarafder, M. Bernien, A. Krüger, Y.-M. Chang, P. M. Oppeneer and W. Kuch, Adv. Mater., 2013, 25, 3473-3477; (k) J. Dreiser, C. Wäckerlin, M. E. Ali, C. Piamonteze, F. Donati, A. Singha, K. S. Pedersen, S. Rusponi, J. Bendix, P. M. Oppeneer, T. A. Jung and H. Brune, ACS Nano, 2014, 5, 4662.

6 J. Klanke, E. Rentschler, K. Medjanik, D. Kutnyakhov, G. Schönhense, S. Krasnikov, I. V. Shvets, S. Schuppler, P. Nagel, M. Merz and H. J. Elmers, Phys. Rev. Lett., 2013, 110, 137202.

7 L. Malavolti, L. Poggini, L. Margheriti, D. Chiappe, P. Graziosi, B. Cortigiani, V. Lanzilotto, F. B. de Mongeot, P. Ohresser, E. Otero, F. Choueikani, P. Sainctavit, I. Bergenti, V. A. Dediu, M. Mannini and R. Sessoli, Chem. Commun., 2013, 49, 11506.

8 (a) T. Santos, J. Moodera, K. Raman, E. Negusse, J. Holroyd, J. Dvorak, M. Liberati, Y. Idzerda and E. Arenholz, Phys. Rev. Lett., 2008, 101, 147201; (b) E. Arenholz, A. Schmehl, D. G. Schlom and G. van der Laan, J. Appl. Phys., 2009, 105, 07E101; (c) A. G. Swartz,
P. M. Odenthal, Y. Hao, R. S. Ruoff and R. K. Kawakami, ACS Nano, 2012, 6, 10063-10069.

9 A. Uldry, F. Vernay and B. Delley, Phys. Rev. B, 2012, 85, 125133.

10 E. Negusse, J. Holroyd, M. Liberati, J. Dvorak, Y. U. Idzerda, T. S. Santos, J. S. Moodera and E. Arenholz, J. Appl. Phys., 2006, 99, 08E507.

11 T. Kataoka, Y. Sakamoto, Y. Yamazaki, V. R. Singh, A. Fujimori, Y. Takeda, T. Ohkochi, S.-I. Fujimori, T. Okane, Y. Saitoh, H. Yamagami and A. Tanaka, Solid State Commun., 2012, 152, 806-809.

12 (a) B. Thole, P. Carra, F. Sette and G. van der Laan, Phys. Rev. Lett., 1992, 68, 1943-1946; (b) P. Carra, B. Thole, M. Altarelli and X. Wang, Phys. Rev. Lett., 1993, 70, 694-697.

13 (a) Y. Teramura, A. Tanaka and T. Jo, J. Phys. Soc. Jpn., 1996, 65, 1053-1055; (b) C. Piamonteze, P. Miedema and F. M. F. de Groot, Phys. Rev. B, 2009, 80, 184410; (c) S. Stepanow, P. Miedema, A. Mugarza, G. Ceballos, P. Moras, J. Cezar, C. Carbone, F. de Groot and P. Gambardella, Phys. Rev. B, 2011, 83, 220401.

14 B. Thole, G. van der Laan, J. Fuggle, G. Sawatzky, R. Karnatak and J.-M. Esteva, Phys. Rev. B, 1985, 32, 5107-5118.

15 A. Lodi Rizzini, C. Krull, T. Balashov, J. J. Kavich, A. Mugarza, P. S. Miedema, P. K. Thakur, V. Sessi, S. Klyatskaya, M. Ruben, S. Stepanow and P. Gambardella, Phys. Rev. Lett., 2011, 107, 177205.

16 (a) P. Wachter, in Handbook on the physics and chemistry of rare earths, ed. K. A. Gschneidner and L. Eyring, North-Holland, New York, USA, 1979, vol. 2, p. 507; (b) J. P. Liu, F. R. de Boer, P. F. de Châtel, R. Coehoorn and K. H. J. Buschow, J. Magn. Magn. Mater., 1994, 132, 159-179; (c) X. Wan, J. Dong and S. Y. Savrasov, Phys. Rev. $B, 2011,83,205201$.

17 C. Piamonteze, U. Flechsig, S. Rusponi, J. Dreiser, J. Heidler, M. Schmidt, R. Wetter, M. Calvi, T. Schmidt, H. Pruchova, J. Krempasky, C. Quitmann, H. Brune and F. Nolting, J. Synchrotron Radiat., 2012, 19, 661-674. 